\title{
A year of Guided OA
}

\author{
The Guided Open Access pilot we are trialling with five other journals in the Nature Portfolio will continue into \\ 2022. We highlight some of the main lessons we've learned so far.
}

A year has passed since we started accepting submissions in the Guided Open Access trial that we developed with Nature Genetics, Nature Methods, Nature Communications, Communications Biology and Communications Physics, so now seems like an appropriate time to take stock.

A reminder: Guided OA offers authors the opportunity to submit their manuscripts for simultaneous consideration at three journals within one of the genetics, methods or physics submission streams (see image), receive an Editorial Assessment Report (EAR) and, in the event of acceptance in one of Nature Genetics, Nature Methods or Nature Physics, obtain a substantial discount on the article processing charge required to make the paper Open Access.

When we announced the initiative ${ }^{1}$, we were acutely aware that this was a lot of information to unpack, especially since we were combining a somewhat abstract and unfamiliar editorial concept with a new publishing model. However, now that we have published our first physics Guided OA articles ${ }^{2-7}$, we are in a position to discuss concrete examples: subject to the authors' permission, the EARs are published alongside the papers as supplementary information.

As these documents hopefully make clear, the EARs provide the authors with help to interpret the feedback from the reviewers, as well as guidance for adhering to best-practice on openness, transparency and reproducibility - just as we envisaged when we set up the trial. However, what also emerges from these early examples is the efficiency made possible from the simultaneous consideration at multiple journals: editors from Nature Physics, Nature Communications and Communications Physics all weigh in on where the paper sits with respect to their editorial criteria, and so authors receive nuanced feedback and options from all three venues.

But what did the authors themselves think about Guided OA? Behind the scenes, we worked closely with our marketing and publishing colleagues to gather their feedback as the pilot progressed. The

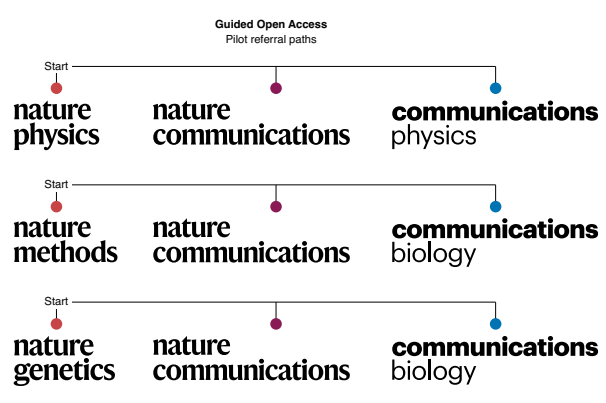

following key lessons have become apparent.

Firstly, authors are positive about the EARs. They found the structured format of the document helpful and especially appreciated the consistency of editorial guidance in the light of the different preferences of the reviewers, as well as time saved as a result of the simultaneous consideration of their work at multiple journals. That being said, these benefits weren't necessarily obvious for many at the point of submission - a reminder that when it comes to messaging, sometimes less is more.

Secondly, we found that authors pay the editorial assessment charge on time. This was a big question about Guided OA when it was first announced, and one that struck a nerve: how would authors respond to viewing journals as an explicit service, rather than just a means to a product (in the form of a published paper)? We knew there were very strong views about this from the outset of the trial. We now know empirically that, when engaged with thoughtfully, many authors find this shift reasonable.

We've also found that Guided OA has been particularly attractive for large-scale consortia and collaborations that tend to produce clusters of papers and follow-up results. While they are still making their way through the pilot at the time of writing, we have a number of excellent submissions under consideration from both genomics and physics consortia, all of which are likely to find a home in one of the pilot's participating journals in the coming weeks and months.
Finally, we've learned that the collaborative approach that Guided OA requires from our journals' editors also works. That's not to say we didn't have to adapt to the challenges posed by the trial - just as Amdahl's law dictates a limit to the speedup provided by adding more processors executing a computer program in parallel, the communication overhead required by an increasing number of highly opinionated editors does not scale favourably. But by thinking carefully about when and how we assign responsibility for editorial tasks within our processes, we have developed them to be considerably more effective than they were when we started the pilot.

While we view the Guided OA trial as a success so far, we are not yet at the point of being able to roll it out to include other journals. In particular, the volume of submissions we have received relative to the ordinary 'business as usual' submission stream has been comparatively low, and so far we've produced about 70 EARs across all the participating journals. We put this down to the novelty of the proposition, and as more papers and EARs come online we hope more authors will become familiar with it and decide to take the plunge.

Our decision is therefore to continue the pilot in its current trial format into 2022 . We can certainly see huge scope for innovation and development in the EAR so that it better reflects the needs of the different disciplines that we serve, for example by supporting initiatives such as code review. We will be sure to report back as we obtain more data and information on author takeup and participation.

Published online: 13 January 2022 https://doi.org/10.1038/s41567-021-01499-3

\footnotetext{
References

1. Nat. Phys. 17, 1 (2021).

2. Pásztor, Á. et al. Nat. Commun. 12, 6037 (2021).

3. Osumi, K. et al. Commun. Phys. 4, 211 (2021).

4. Hibino, Y. et al. Nat. Commun. 12, 6254 (2021).

5. Barfknecht, R. E. et al. Commun. Phys. 4, 252 (2021).

6. Speirs, N. B. et al. Nat. Commun. 12, 7250 (2021).

7. Sekiguchi, Y. et al. Commun. Phys. 4, 264 (2021).
} 